\title{
ON INTEGRATION IN QUASI-BANACH SPACES OF SEQUENCES
}

\author{
A. V. Keller ${ }^{1}$, alevtinak@inbox.ru, \\ A. A. Zamyshlyaeva ${ }^{1}$, alzama@mail.ru, \\ M. A. Sagadeeva ${ }^{1}$, sam79@74.ru \\ ${ }^{1}$ South Ural State University, Chelyabinsk, Russian Federation
}

\begin{abstract}
Since a quasi-Banach space of sequences named quasi-Sobolev space is not locally convex it is not possible to speak about integrability of each continuous function. The main aim of this work is to get conditions sufficient for existence of Riemann integral for the function with values in such space. We use the properties of metrizability and local pseudoconvexivity of the space to show the existence of integral for an analytic function.

Besides the introduction and bibliography, the article includes two sections. In the first section the mentioned properties of quasi-Banach spaces are discussed. In the second section we obtain the conditions for integration of function with values in quasi-Banach spaces of sequences.
\end{abstract}

Keywords: quasi-Banach spaces; quasi-Sobolev spaces; analytic vector-function; Riemann integral.

\section{Introduction}

Apparently quasi-Banach spaces appeared simultaneously with Banach spaces. The study of Banach spaces raises a natural question about, for example, the structure of the space of sequences $\ell_{q}$ for $0<q<1$. However, due to the nature of such spaces they have been for a long time treated by researchers as special narrow types of spaces. Currently, however, interest in such spaces has increased (see, e.g. $[1,2,3,4,5,6]$ ). Moreover, this interest has both theoretical $[1,2,7]$ and practical nature [3, 5].

When solving problems arising in practical applications $[3,5]$ one needs to use the notion of integrability in quasi-Banach spaces. The Mazur and Orlicz theorem $[8$, Theorem 3.5.1] on Riemann integrability for continuous function is equivalent to the local convexity of the space, where the integrand function takes values. It is a well known fact that a quasi-normed space is metrizable [7, Lemma 3.10.1], but in general it is not locally convex [8, Sec. 3.1]. Therefore continuous functions might be nonintegrable and the problem of integrability in quasi-Banach spaces can be solved with additional conditions on the integrand function and the space it takes values in.

The aim of this paper is to establish the conditions allowing to consider the integrals for functions with values in quasi-Banach spaces. In considering this question we use the results of the monograph by $\mathrm{S}$. Rolewicz [8].

The article consists of introduction and two parts. The first one includes the necessary terminology to justify the issue. And in the second part we substantiate the existence of the Riemann integral in quasi-Banach spaces.

\section{Locally Pseudoconvex Spaces}

Recall the standard notation and terminology from $[7,8]$. Let $\mathfrak{U}$ be a some linear space with scalars from $\mathbb{R}$. We say that ordered pair $(\mathfrak{U} ; \mathfrak{U}\|\cdot\|)$ is a quasi-normed space if 
(i) $\forall u \in \mathfrak{U}_{\mathfrak{U}}\|u\| \geq 0$ and $\mathfrak{U}\|u\|=0 \Leftrightarrow u=\mathbf{0}$, where $\mathbf{0} \in \mathfrak{U}$;

(ii) $\forall u \in \mathfrak{U} \forall \alpha \in \mathbb{R}_{\mathfrak{U}}\|\alpha u\|=|\alpha| \cdot \mathfrak{U}\|u\|$;

(iii) $\forall u, v \in \mathfrak{U} \quad \mathfrak{U}\|u+v\| \leq C(\mathfrak{u}\|u\|+\mathfrak{u}\|v\|)$, where constant $C \geq 1$ is independent from $u$ and $v$.

If $C \geq 1$ then the function $\mathfrak{u}\|\cdot\|: \mathfrak{U} \rightarrow \overline{\mathbb{R}}_{+}$is called a quasi-norm. If $C=1$ then the function $\mathfrak{U}\|\cdot\|: \mathfrak{U} \rightarrow \overline{\mathbb{R}}_{+}$is a norm. So, quasi-normed space is a generalization of the normed space. Here and below we denote the quasi-normed space $(\mathfrak{U} ; \mathfrak{U}\|\cdot\|)$ by $\mathfrak{U}$ for brevity.

The quasi-norm $\mathfrak{u}\|\cdot\|$ determines a topology on $\mathfrak{U}$ by natural way. Basis of neighborhoods contains all sets, which are defined by $\{u \in \mathfrak{U}: \mathfrak{U}\|u-v\|<\varepsilon\}$, where $\varepsilon \in \mathbb{R}_{+}$. If $C=1$ then this topology is determined by metric $\rho(u, v)=\mathfrak{u}\|u-v\|$. However, if $C>1$ then the quasi-normed space is a metric space too.

Lemma 1. [7, Lemma 3.10.1] Let $\mathfrak{U}$ be a quasi-normed space and $p \in \mathbb{R}$ be defined by equality $(2 C)^{p}=2$. Then there exists a metric $d: \mathfrak{U} \times \mathfrak{U} \rightarrow \mathbb{R}$ on $\mathfrak{U}$ such that for all $u \in \mathfrak{U}$ inequality

$$
d(\mathbf{0}, u) \leq \mathfrak{u}\|u\|^{p} \leq 2 d(\mathbf{0}, u)
$$

holds.

By this Lemma we have notions of fundamental sequence $\left\{u_{n}\right\} \subset \mathfrak{U}$ : $\lim _{n, l \rightarrow \infty} \mathfrak{U}\left\|u_{n}-u_{l}\right\|=0$, completeness of space and convergence of a series in quasi-Banach space, see [7, Lemma 3.10.2]. A complete quasi-normed space is called quasi-Banach.

Example 1. Let $\left\{\lambda_{k}\right\} \subset \mathbb{R}_{+}$be a monotonic sequence such that $\lim _{k \rightarrow \infty} \lambda_{k}=+\infty$, and $p \in \mathbb{R}_{+}$. Put

$$
\ell_{p}^{m}=\left\{u=\left\{u_{k}\right\} \subset \mathbb{R}: \sum_{k=1}^{\infty}\left(\lambda_{k}^{\frac{m}{2}}\left|u_{k}\right|\right)^{p}<+\infty\right\} .
$$

Lineal $\ell_{p}^{m}$ for all $m \in \mathbb{R}, p \in \mathbb{R}_{+}$with a quasi-norm of element $u=\left\{u_{k}\right\} \in \ell_{p}^{m}$

$$
{ }_{p}^{m}\|u\|=\left(\sum_{k=1}^{\infty}\left(\lambda_{k}^{\frac{m}{2}}\left|u_{k}\right|\right)^{p}\right)^{1 / p}
$$

is a quasi-Banach space (for $p \in[1,+\infty)$ it is a Banach space). Note that if $p \in(0,1)$, then constant $C=2^{1 / p}$. The spaces $\ell_{p}^{m}$ are called quasi-Sobolev in [6].

Now let $(\mathfrak{U}, \mathrm{d})$ be a metric space. A set $A \subset \mathfrak{U}$ is said to be a starlike (starshaped) set if $\{t u \in \mathfrak{U}: u \in A\} \equiv t A \subset A$ for all $t \in(0,1]$. Define $\{u+v \in \mathfrak{U}: u, v \in A\} \equiv A+A$. The modulus of concavity of a starlike set $A$ is defined by

$$
c(A)=\inf \{s>0: A+A \subset s A\}
$$

with the convention that the infimum of empty set is equal to $+\infty$. A starlike set $A$ with the finite modulus of concavity, $c(A)<+\infty$, is called pseudoconvex.

A metric space $\mathfrak{U}$ is called locally pseudoconvex if there is a basis of neighborhoods of zero $\left\{U_{n}\right\}$ which are pseudoconvex. If moreover $c\left(U_{n}\right) \leq 2^{1 / p}$, we say that the space $\mathfrak{U}$ is called locally p-convex. 
Theorem 1. [8, Corollary 3.4.6] The space $\ell_{p}^{m}, 0<p \leq 1$ is locally p-convex with $c\left(\ell_{p}^{m}\right)=2^{1 / p}$

\section{Integration of Functions with Values in Quasi-Banach Spaces}

Let $f(t)$ be a function defined on an interval $[a, b]$ with values in quasi-Banach space $\mathfrak{U}$. As in classical definition of the Riemann integral, we define a normal sequence of subdivisions of interval $[a, b]$ as a sequence of subdivisions

$$
a=t_{0}^{n}<t_{1}^{n}<\ldots<t_{k(n)}^{n}=b,
$$

such that $\lim _{n \rightarrow \infty} \sup _{1 \leq i \leq k(n)}\left|t_{i}^{n}-t_{i-1}^{n}\right|=0$.

We say that a function $f(t)$ is Riemann integrable if, for each normal sequence of subdivisions of the interval $[a, b]$ and arbitrary $s_{i}^{n} \in\left(t_{i-1}^{n}, t_{i}^{n}\right)$, there exists a limit of the sum

$$
\sum_{i=1}^{k(n)} f\left(s_{i}^{n}\right)\left(t_{i}^{n}-t_{i-1}^{n}\right) .
$$

As in the calculus, one may show that this limit does not depend on the sequence of subdivisions. It will be called the Riemann integral of the function $f(t)$, and we shall write it as

$$
\int_{a}^{b} f(t) d t
$$

It is easy to verify that the Riemann integral defined as above has the similar arithmetical properties to those it has in the calculus. Namely, if $f(t)$ and $g(t)$ are integrable functions (i.e. such that the Riemann integrals exist), then the sum $f(t)+g(t)$ is integrable and

$$
\int_{a}^{b}(f(t)+g(t)) d t=\int_{a}^{b} f(t) d t+\int_{a}^{b} g(t) d t,
$$

if a function $f(t)$ is integrable, then for any scalar $K$ the function $K f(t)$ is integrable and

$$
\int_{a}^{b} K f(t) d t=K \int_{a}^{b} f(t) d t
$$

if a function $f(t)$ is integrable on $[a, b]$, then for each $c \in(a, b)$, the function $f(t)$ is integrable on intervals $[a, c]$ and $[c, b]$ and

$$
\int_{a}^{b} f(t) d t=\int_{a}^{c} f(t) d t+\int_{c}^{b} f(t) d t .
$$

We say that a function $f(t)$ with values in quasi-Banach space $\mathfrak{U}$ is analytic if, for any $t_{0}$, there is a neighborhood $U$ of a point $t_{0}$ such that, for $t \in U$, the function $f(t)$ can be 
represented by a power series

$$
f(t)=\sum_{n=0}^{\infty}\left(t-t_{0}\right)^{n} f_{n}, \quad f_{n} \in \mathfrak{U} .
$$

Theorem 2. Let $\mathfrak{U}=\ell_{p}^{m}$ with $0<p \leq 1$. Then all analytic function $f(t)$ defined on an interval $[a, b]$ with values in $\mathfrak{U}$ are integrable.

Proof.

By Lemma $1 \mathfrak{U}=\ell_{p}^{m}$ is metrizable and by Theorem 1 it is locally $p$-convex. Therefore all conditions of Theorem 3.5.2 [8] are fulfilled and the statement is true.

Let $\mathbb{C}$ be a complex plane. Let $D$ be an open set in $\mathbb{C}$. Let $f(t)$ be a function defined on $D$ with values in the quasi-Banach space $\mathfrak{U}$. In the same way as for the function of the real argument, we say that $f(t)$ is analytic in $D$, if for each $z_{0} \in D$, there is a neighbourhood $U\left(z_{0}\right)$ of a point $z_{0}$ such that the function $f(t)$ can be represented in $U\left(z_{0}\right)$ as a sum of power series

$$
f(z)=\sum_{n=0}^{\infty}\left(z-z_{0}\right)^{n} f_{n}, \quad f_{n} \in \mathfrak{U} .
$$

As in the real-argument case, we can define the Riemann integral of a function $f(t)$ on a curve contained in $D$. By the analogy with Theorem 2 we have next

Theorem 3. Let $\mathfrak{U}=\ell_{p}^{m}$ with $0<p \leq 1$. Let $D$ be an open set in $\mathbb{C}$ and $\Gamma$ be a smooth curve contained in $D$. Then for all analytic function $f(z)$ defined on $D$ with values in $\mathfrak{U}$ the integral $\int_{\Gamma} f(z) d z$ exists.

Using the same arguments as in the classical theory of analytic functions, we can obtain

Theorem 4. Let $\mathfrak{U}=\ell_{p}^{m}$ with $0<p \leq 1$. Let $D$ be an open set in $\mathbb{C}$ and $\Gamma$ be a smooth curve contained in $D$. Let $f(z)$ be an analytic function in $D$. Then

$$
\begin{gathered}
\int_{\Gamma} f(z) d z=0 \\
f\left(z_{0}\right)=\frac{1}{2 \pi i} \int_{\Gamma} \frac{f(z)}{z-z_{0}} d z
\end{gathered}
$$

for each $z_{0}$ inside the domain surrounded by $\Gamma$,

$$
f^{(n)}\left(z_{0}\right)=\frac{n !}{2 \pi i} \int_{\Gamma} \frac{f(z)}{\left(z-z_{0}\right)^{n+1}} d z
$$

where $z_{0}$ and $\Gamma$ are the same as above. 


\section{References}

1. Aleksandrov A.B. [Quasi-Normed Spaces in Compex Analisys]. ScD (Math) Work. Leningrad, 1983. (in Russian)

2. Kalton N.J. Quasi-Banach spaces. Handbook of the Geometry of Banach Spaces, 2003, vol. 2, pp. 1099-1130.

3. Candes E., Romberg J., Tao T. Time-frequency analysis on modulation spaces $M_{m}^{p, q}$, $0<p, q<\infty$. Applied and Computational Harmonic Analysis, 2004, vol. 16, issue 1, pp. $1-18$.

4. Rauhut H. Wiener amalgam spaces with respect to quasi-Banach spaces. Colloquium Mathematicum, 2007, vol. 109, no. 2, pp. 345-362.

5. Vovk S.M., Borulko V.F. Statement of a problem of definition of linear signals parameters in quasinormed space. Radioelectronics and Communications Systems, 2010, vol. 53, no. 7, pp. 367-375. doi: 10.3103/S0735272710070046

6. Keller A.V., Al-Delfi J.K. Holomorphic Degenerate Groups of Operators in QuasiBanach Spaces. Bulletin of the South Ural State University. Series "Mathematics. Mechanics. Physics", 2015, vol. 7, no. 1, pp. 20-27. (in Russian)

7. Bergh J., Löfström J. Interpolation Spaces. An Introduction. Berlin, Heidelberg, New York, Springer-Verlag, 1976.

8. Rolewicz S. Metric Linear Spaces. Warsaw, PWN, 1985.

Alevtina V. Keller, Doctor of Physico-Mathematical Sciences, Associate Professor, Dean of the Faculty of Mathematics, Mechanics and Computer Science, South Ural State University, Chelyabinsk, Russian Federation, alevtinak@inbox.ru.

Alyona A. Zamyshlyaeva, Doctor of Physico-Mathematical Sciences, Associate Professor, Department of Equation of Mathematical Physics, Mechanics and Computer Science, South Ural State University, Chelyabinsk, Russian Federation, alzama@mail.ru.

Minzilya A. Sagadeeva, Candidate of Physico-Mathematical Sciences, Associate Professor, Head of Department of Mathematical Modeling, South Ural State University, Chelyabinsk, Russian Federation, sam79@74.ru.

Received February 18, 2015 